\title{
O diamino fluoreto de prata no controle da cárie na primeira infância durante a pandemia da COVID-19
}

The silver diamine fluoride in the management of early childhood caries during COVID-19 pandemic

Uso de fluoruro diamino de plata en el control de caries en la primera infancia durante la pandemia del COVID-19

Recebido: 23/04/2021 | Revisado: 03/05/2021 | Aceito: 07/05/2021 | Publicado: 21/05/2021

\author{
Emerson Tavares de Sousa \\ ORCID: https://orcid.org/0000-0002-5368-8721 \\ Universidade Estadual de Campinas, Brasil \\ E-mail: etavaresodonto@gmail.com. \\ Camila Lopes Crescente \\ ORCID: https://orcid.org/0000-0001-8002-6072 \\ Universidade Estadual de Campinas, Brasil \\ E-mail: micrescente@hotmail.com \\ Aline Laignier Yoshikawa \\ ORCID: https://orcid.org/0000-0002-5788-2070 \\ Universidade Estadual de Campinas, Brasil \\ E-mail: alaignier@yahoo.com.br \\ Marinês Nobre dos Santos \\ ORCID: https://orcid.org/0000-0002-2120-3187 \\ Universidade Estadual de Campinas, Brasil \\ E-mail: nobre@fop.unicamp.br
}

\begin{abstract}
Resumo
Objetivo: Abordar aspectos de interesse clínico relacionados ao uso do diamino fluoreto de prata (DFP) no controle da cárie na primeira infância (CPI) durante a pandemia da COVID-19. Métodos: A busca para revisão de literatura foi executada na base de dados PubMed. Os descritores foram escolhidos pela importância na delimitação do tema e a síntese da literatura foi realizada de acordo com os tópicos: (1) efetividade clínica, indicações e limites do DFP e (2) uso clínico do DFP no contexto da COVID-19. Resultados: A pandemia da COVID-19 afeta todos os setores da sociedade, e, especialmente para o odontopediatra, impõe desafios em um cenário de difícil tomada de decisão e passível de contaminação. O tratamento da doença cárie com DFP ganha uma conotação importante pela sua eficácia na paralização da atividade de cárie, prevenção de novas lesões, segurança, facilidade de uso e baixo custo. Além disso, o DFP é considerado uma terapia minimamente invasiva que dispensa uso de instrumentos rotatórios (que tem alto potencial contaminante via dispersão de aerossol), e reduz complicações como pulpite e perda dentária e, portanto, o número de consultas. O DFP é também uma opção clínica na qual há uma maior aceitação comportamental pela criança. Como ponto negativo, o DFP pode causar insatisfação estética devido ao escurecimento da superfície afetada. Conclusão: O controle de lesões de cárie em crianças com CPI utilizando DFP, durante a pandemia da COVID-19, é uma opção eficaz, barata, de fácil aplicação e que oferece menos riscos de contaminação pelo coronavírus.
\end{abstract}

Palavras-chave: Pré-escolares; Cárie dentária; Cariostáticos; Síndrome respiratória aguda grave; SARS-CoV.

\begin{abstract}
Objective: To address aspects of clinical interest related to the use of silver diamine fluoride (SDF) in the management of early childhood caries (ECC) during the COVID-19 pandemic. Methods: The search for the literature review was carried out in the PubMed database. The descriptors were chosen according to their importance in delimiting the theme and the literature synthesis considered the topics: (1) clinical effectiveness, indications, and limits of SDF, and (2) clinical use of SDF in COVID-19 context. Results: COVID-19 pandemic affects all sectors of society, and especially for pediatric dentists it imposes challenges in a scenario of difficult decision-making and liable of contamination. The treatment of caries disease with SDF gains an important connotation due to its effectiveness in the arrestment and prevention of caries lesions, safety, ease use, and low cost. It is considered a minimally invasive therapy that dispenses the use of rotary instruments (which have a high potential for contamination via aerosol dispersion) and reduces complications such as pulpitis and tooth loss and, therefore, the number of appointments. Besides, SDF is an option in which there is greater behavioral acceptance by the child. As a negative point, SDF can cause aesthetic dissatisfaction due to the darkening of the affected tooth surface. Conclusion: The caries lesions
\end{abstract}


control in children with ECC using SDF, during the COVID-19 pandemic, is an effective, inexpensive, and easy to apply option. In addition, it offers a lower contamination risk by the coronavirus.

Keywords: Child preschool. Dental caries; Cariostatic agents; Severe acute respiratory syndrome; SARS virus.

\section{Resumen}

Objetivo: Abordar aspectos de interés clínico relacionados con el uso de fluoruro diamino de plata (DFP) en el control de la caries en la primera infancia (CPI) durante la pandemia del COVID-19. Métodos: La búsqueda se realizó en la base de datos PubMed. Los descriptores fueron elegidos de acuerdo con el tema y la síntesis de la literatura basado en: (1) efectividad clínica, indicaciones y limitaciones de DFP; (2) uso clínico de DFP en el contexto del COVID-19. Resultados: La pandemia del COVID-19 afecta a todos los sectores de la sociedad, en especial a los odontopediatras, imponiendo desafíos por ser un escenario de difícil toma de decisiones. El tratamiento de la enfermedad de caries con DFP adquiere una connotación importante por su eficacia para detener su actividad, prevenir nuevas lesiones, seguridad, facilidad de uso y bajo costo. Además, se considera una terapia mínimamente invasiva al no requerir el uso de instrumentos rotativos (presentan un alto potencial de contaminación por dispersión de aerosoles). Este método reduce complicaciones como pulpitis y pérdida dentaria, por tanto, el número de consultas, además, de ser una opción clínica en la que existe una mayor aceptación conductual por parte del niño. Como punto negativo, DFP puede causar insatisfacción estética debido al oscurecimiento de la superficie afectada. Conclusión: El control de las lesiones de caries en niños con IPC mediante DFP, durante la pandemia de COVID-19, es una opción eficaz, económica y fácil de aplicar que ofrece un menor riesgo de contaminación por esta enfermedad.

Palabras clave: Preescolar; Caries dental; Cariostáticos; Síndrome respiratorio agudo grave; Virus del SRAS.

\section{Introdução}

A alta taxa de transmissibilidade e mortalidade da Síndrome Respiratória Aguda Grave Coronavírus 2 (SARS-CoV-2) ou infecção por coronavírus 2019 (COVID-19) tem modificado a dinâmica mundial no sentido de conter a disseminação do vírus como forma de evitar a saturação dos sistemas de saúde enquanto que o torna mais hábil para o controle clínico da doença (Matrajt e Leung, 2020). Nesse cenário pandêmico e de emergência para diversos países, inclusive para o Brasil, uma das medidas de maior impacto é o distanciamento social (Aquino et al., 2020; Kissler et al., 2020).

Desde o primeiro caso da COVID-19 no Brasil em 26 de fevereiro de 2020, diversos estados entraram em alerta e incorporaram esse modelo de mitigação da infecção, que coaduna com a recomendação da Organização Mundial da Saúde (OMS, 2020). Apesar do distanciamento social ser uma medida de saúde pública importante, ainda há incertezas quanto ao tempo de confinamento necessário para evitar a infecção em larga escala, ao mesmo tempo que evoca o redesenho de situações cotidianas em todos os espaços sociais em adequação ao "novo normal”. Essas adaptações são ainda mais relevantes se considerarmos evidências que sugerem a necessidade de isolamento social até 2022 ou mesmo o ressurgimento do contágio por COVID-19 até 2024 (em caso de eliminação aparente) (Kissler et al., 2020).

Respeitando as recomendações de distanciamento social e considerando o alto risco de contágio por COVID-19 que o ambiente odontológico impõe, diversos órgãos internacionais (Academia Americana de Odontopediatria, Academia Europeia de Odontopediatria e Academia Latinoamericana de Odontopediatria) e nacional (Academia Brasileira de Odontopediatria) restringiram a indicação de procedimentos clínicos odontopediátricos para casos de urgência. Durante meses houve uma inibição do acesso à serviços odontológicos no setor público e privado e é provável que o cenário pós-pandêmico traga consigo o desafio de lidar com uma maior demanda por serviços e limitação de recursos devido ao colapso econômico desencadeado pela COVID-19 (Garrett, 2020). Nesse contexto, é importante considerar que dentre todas as doenças bucais na primeira infância, a cárie é o problema mais prevalente e que causa mais consequências biopsicossociais na vida das crianças (Tinanoff et al., 2019; Pitts et al., 2019). Além disso, a CPI é responsável por um aumento da demanda por serviços cada vez mais especializados e gastos com o tratamento (Casamassimo et al., 2009).

Na clínica infantil, a pandemia da COVID-19 impõe desafios para o odontopediatra em um cenário de difícil tomada de decisão e medo de contaminação. Como um fenômeno social, apesar da oportunidade de coesão familiar que o isolamento social pode trazer, o sentimento de medo e ameaça imputados pela resposta à pandemia podem desencadear o esgotamento 
emocional na criança e em seus pais (Bavel et al., 2020; Fegert et al., 2020). Este fato pode acarretar um impacto na percepção sobre saúde bucal no sentido de torná-la menos importante em um cenário de crise ou tornar mais difícil a adesão de práticas saudáveis. Ainda, o estresse, depressão e ansiedade vivenciados nesse momento podem ser fatores que dificultam o tratamento da CPI que muitas vezes já é complexo. Em relação a contaminação, o uso de instrumentos rotatórios no consultório e seu potencial impacto da disseminação do vírus via aerossol e gotas de saliva e sangue (Meng et al., 2020; Luzzi et al., 2020) imprimem a sensação de insegurança e sinaliza para a redução do uso desses instrumentos no momento atual.

Nessas circunstâncias, coincidente com o advento da filosofia do minimamente invasivo (Schwendicke et al., 2019), o manejo de lesões de cárie ativas em dentina (ICDAS 4, 5 ou 6 - sem comprometimento pulpar) pode ser realizado por meio do tratamento restaurador atraumático (TRA), de coroas metálicas pré-formadas pela técnica de Hall ou do cariostático (diamino fluoreto de prata - DFP). Em lesões em esmalte (ICDAS 1,2 ou 3), a aplicação profissional de fluoreto, resinas infiltrantes, verniz, selantes de fóssulas e fissuras, assim como também do diamino fluoreto de prata são indicados (Tinanoff et al., 2019). Idealmente, valendo-se da premissa de que esses procedimentos isoladamente não paralisam a doença, o controle dietético e o uso de dentifrício fluoretado são indispensáveis. Em época de pandemia a modalidade de aconselhamento remoto é uma opção plausível para evitar complicações de lesões que irão, deliberadamente, estimular a necessidade de cuidados mais complexos.

O uso do diamino fluoreto de prata (Duangthip et al., 2016; Gao et al., 2016; Chibinski et al., 2017) ganha especial atenção por sua eficácia clínica na paralização de lesões, na prevenção da progressão da doença e por ser uma medida de controle que não oferece danos à estrutura dental hígida ou cariada, de baixo custo e ainda, que apresenta segurança e facilidade na aplicação não sendo necessária a utilização de instrumentos rotatórios ou mesmo de infraestrutura sofisticada (Mei et al., 2016; Oliveira et al., 2019). Portanto, considerando os aspectos envolvidos no manejo clínico dos pacientes com CPI durante a pandemia da COVID-19, essa revisão de literatura objetivou abordar aspectos de interesse clínico relacionados ao uso do diamino fluoreto de prata no controle da cárie na primeira infância.

\section{Metodologia}

O estudo trata-se de uma revisão de literatura realizada por meio da busca de artigos na base de dados Public Medline (PubMed). Os descritores foram escolhidos por sua importância na delimitação do tema de acordo com termos do Medical Subject Headings (MeSH): preschoolers, dental caries, cariostatic agents, Coronavirus or COVID-19, e silver diamine fluoride. O termo early childhood caries, apesar de não constar no $M e S H$, foi incluído na busca.

Os dados foram selecionados de acordo com os seguintes critérios: trabalhos completos em português ou inglês e que estivessem de acordo com a narrativa do tema. Para a escolha foram considerados os seguintes tipos de artigos: revisão de literatura, revisão sistemática, casos clínicos e trabalhos de pesquisa; foram incluídos todos os artigos disponíveis até o ano de 2020.

A síntese da literatura foi realizada de acordo com os tópicos: (1) mecanismo de ação (2) efetividade clínica, indicações e limites do DFP e (3) Uso clínico do DFP no contexto da COVID-19.

\section{Resultados e Discussão}

\subsection{Diamino Fluoreto De Prata: mecanismo de ação}

O DFP é um sal $\left(\mathrm{Ag}\left(\mathrm{NH}_{3}\right)_{2} \mathrm{~F}\right)$ que contém em sua formulação íons prata e fluoreto que formam um complexo com a amônia. Atualmente, não existe um consenso a respeito do seu mecanismo de ação na dinâmica multifatorial do processo de cárie; contudo, tem sido sugerido que o DFP promove: aumento da remineralização e diminuição da desmineralização pela ação do fluoreto na formação de fluorapatita e fluoreto de cálcio, preservação do colágeno presente na dentina por meio da inibição das metaloproteinases e redução do crescimento e a adesão de bactérias pela ação da prata (Rosenblatt et al., 2009; 
Mei et al., 2016; Zhao et al. 2018).

O DFP, quando em contato com a superfície dental cariada, reage com a hidroxiapatita $(\mathrm{Ca} 10(\mathrm{PO} 4) 6(\mathrm{OH}) 2)$ do esmalte e da dentina. Esse processo ocorre em ambiente básico e culmina na formação de produtos como fluoreto de cálcio, fosfato de prata e amoníaco monohidratado (Rosenblatt et al., 2009). Tal reação é mostrada na Figura 1 seguir:

Figura 1: Reação da hidroxiapatita com a solução de diamino fluoreto de prata em meio básico resultando em produtos como fluoreto de cálcio, fosfato de prata e amoníaco monohidratado.

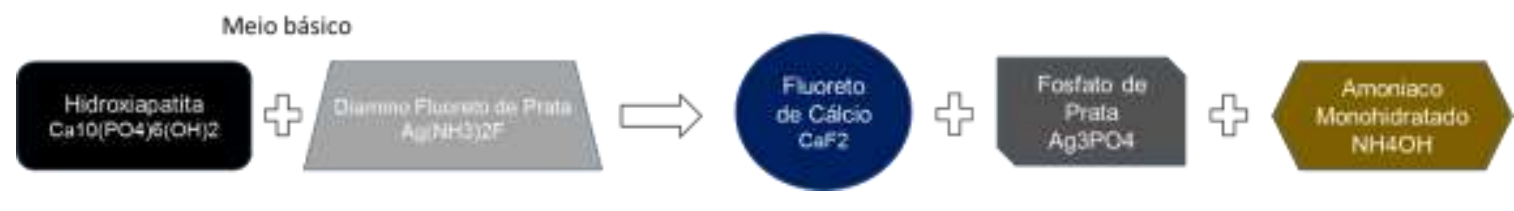

Fonte: Autores.

O fluoreto de cálcio, produto da reação anterior, em ambiente ácido é dissociado em íons cálcio e flúor. Como mostrado na Figura 2:

Figura 2: Reação em meio ácido da dissociação do fluoreto de cálcio em ions cálcio e fluor.

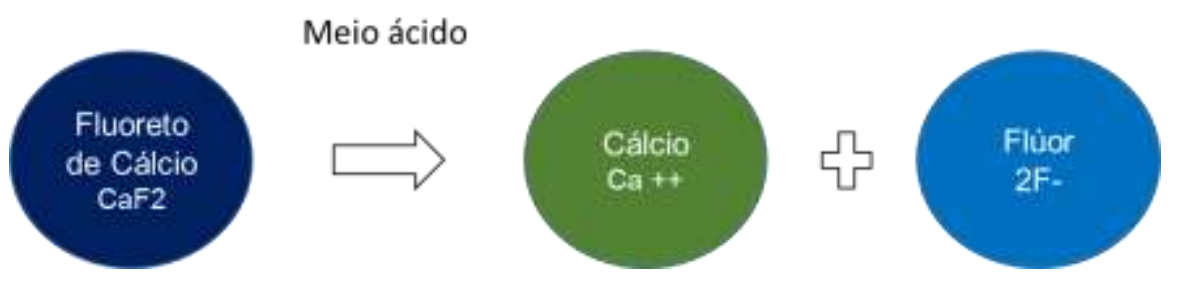

Fonte: Autores.

Por fim, a hidroxiapatita presente no esmalte e dentina dental liga-se aos íons flúor disponíveis e formam a fluorapatita (Ca10(PO4)6F2). Expressa pela seguinte reação (Figura 3):

Figura 3: Reação da hidroxiapatita com o fluoreto para formar a fluorapatita.

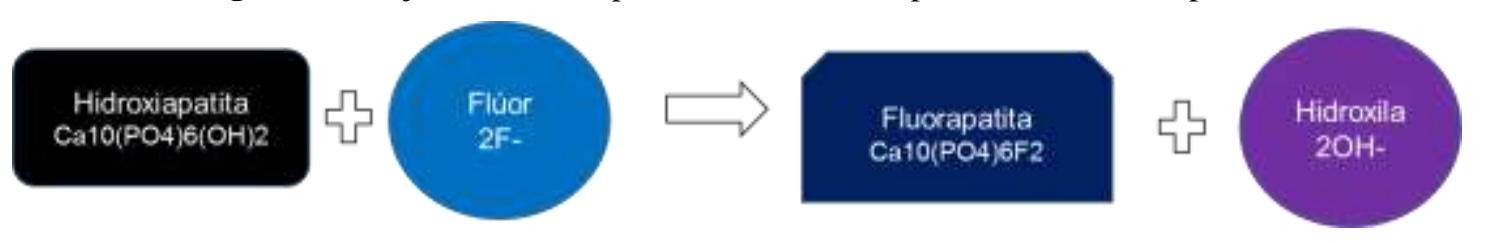

Fonte: Autores.

No estudo de Suzuki et al., (1974) foi demonstrado que após a aplicação do diamino fluoreto de prata a 38\%, o fluoreto penetrou em até $20 \mu \mathrm{m}$ no esmalte; a prata, por sua vez, foi detectada à $10 \mu \mathrm{m}$ da superfície do esmalte. Além disso, esse mesmo estudo identificou que o fluoreto de cálcio e o fosfato de prata formados são eliminados num período de 10 dias e uma semana, respectivamente. Modelos experimentais in vitro em que o DFP a 38\% foi aplicado e os espécimes de esmalte foram submetidos a desafios cariogênicos, demonstraram que a profundidade da lesão de cárie formada foi menor quando comparada a um controle negativo (Klein et al., 1999; Rosas et al., 2014).

Na superfície da dentina desmineralizada, foi observado que após a aplicação do DFP a 29\% e subsequente desafio cariogênico (biofilme + sacarose), a dentina tratada apresentava menor perda de cálcio e fósforo e maior absorção de flúor que 
o grupo controle (Knight et al., 2009). Utilizando uma concentração maior (DFP a 38\%), Chu et al., (2012) e Mei et al., (2012) encontraram resultados similares. No estudo de Mei et al. (2012) espécimes de dentina tratados com DFP a 38\% tiveram a perda mineral de lesões cariosas reduzida após um desafio cariogênico biológico (biofilme) de 7 dias.

Em relação ao poder de penetração do material e sua capacidade de atuar como um agente ativo na prevenção da progressão da lesão de cárie, foi comprovado que espécimes de dentina tratados com DFP a 38\% apresentavam profundidade de lesão reduzida (Mei et al., 2012) e uma camada altamente mineralizada de cerca de $150 \mu \mathrm{m}$ (Mei et al., 2014). Além disso, o estudo de Chu e Lo (2008) concluiu que após a aplicação de DFP a 38\% em aplicações anuais, a microdureza foi mais alta na dentina superficial $(50 \mu \mathrm{m})$, porém, em distâncias superiores a $200 \mu \mathrm{m}$ da superfície da lesão, não houve diferença entre a dentina escurecida (crônica) pelo tratamento e a dentina com cárie ativa. Esses resultados sugerem que a remineralização promovida pelo material ajuda a melhorar as propriedades físicas da dentina até uma certa profundidade $(200 \mu \mathrm{m})$.

A dentina é composta por $10 \%$ de água, $20 \%$ de matriz orgânica e os outros $70 \%$ de mineral. A matriz orgânica equivale a $90 \%$ de colágeno do tipo I e os outros $10 \%$ equivalem à matriz de origem não colagênica. Estudos recentes têm demonstrado os efeitos do DFP na composição orgânica da dentina. Esses estudos foram desenvolvidos por uma equipe chinesa (Mei et al., 2012) e são focados na inibição da degradação da matriz de colágeno pela ação do DFP. Primeiro, os autores demonstraram que a concentração de 38\% reduz de forma significativa a degradação do colágeno pelas metaloproteinases (MMPs) quando comparada com as concentrações de 30 e 12\% (Mei et al., 2012). Em uma condição experimental diferente, na qual os espécimes de dentina eram expostos a desafios cariogênicos (biofilme + sacarose) e tratados com DFP a 38\%, foi concluído que o grupo tratado apresentava mais colágeno I intacto (Mei et al., 2012). Além disso, a degradação do colágeno por catepsinas foi menor no grupo tratado com DFP que nos demais grupos, tratados com nitrato de prata (AgNO3) ou fluoreto de sódio (NaF). Esse efeito mediado pelo DFP não foi concentração dependente.

As metaloproteinases presentes na saliva e na matriz orgânica da dentina são ativadas em meio ácido na dentina cariada. A MMP-8 (colagenases de neutrófilos) corta os tipos de colágeno intersticial I, II e III, e é capaz de digerir outras moléculas de matriz extracelular e de matriz não extracelular. A MMP-2 (gelatinase A) e a MMP-9 (gelatinase B) não só degradam as moléculas de colágeno desnaturado como o colágeno tipo IV, mas também outras proteínas em menor grau. A catepsina-B se divide nas extensões de coloéctos não helicoidais de colágeno, e a Cathepsin-K pode catabolizar o colágeno e degradar a dentina (Obermajer et al., 2008; Mazzoni et al., 2015).

O fosfato de prata (Ag3PO4), produto da reação do DFP com a hidroxiapatita, reage com os grupamentos de amino dos aminoácidos bacterianos e nos grupos de tiol dos ácidos nucléicos bacterianos para formar aminoácidos e ácidos nucléicos de prata (Rosenblatt, et al., 2009).

Após a reação da prata com o DNA bacteriano (ácido nucléico) e com as proteínas (aminoácidos), ocorre uma modificação no metabolismo bacteriano que culmina na inibição da replicação do DNA bacteriano, da respiração celular procariótica, da síntese da parede celular e da divisão celular bacteriana. Dessa forma, ocorre um processo de morte celular das bactérias cariogênicas, além da inibição da formação do biofilme (Rosenblatt, et al., 2009).

Em relação aos efeitos do diamino fluoreto de prata na bactéria cariogênica, foi demonstrado que em um biofilme formado com S. mutans, o diamino fluoreto de prata mostrou uma excelente ação antibacteriana a uma concentração inibitória mínima de $0,12 \mu \mathrm{mol} / \mathrm{mL}$. Além disso, o DFP inibiu completamente a aglutinação de $S$. mutans induzida por dextrano a uma concentração de $0,59 \mu \mathrm{mol} / \mathrm{mL}$ (Suzuki et al., 1976). O DFP também promoveu uma inibição do metabolismo bacteriano do $S$. mutans que pode ser observado nas concentrações de 29\% (Knight et al., 2005), 12 e 30\% (de Almeida et al., 2011). Resultados de inibição bacteriana em espécies como S. mutans, S. oralis e L. casei nas concentrações de 12\%, $16 \%$ e $30 \%$ demonstram o ampliado espectro do diamino fluoreto de prata. Esses achados evidenciam a potência antibiótica do material em condições experimentais. 
Estudos realizados em dentina humana demonstraram que após o tratamento com diamino fluoreto de prata, espécimes de dentina humana apresentam menores contagens de colônias e também um número maior de bactérias mortas quando comparados ao grupo controle. Esses resultados são significativos em modelos de biofilme composto por $S$. mutans $e$ A. naeslundii, S. mutans e L. acidophilus, (Mei et al., 2013) além de modelos de biofilme complexo formado por S. mutans, $S$. sobrinus, L. acidophilus, L. rhamnosus e A. naeslundii (Mei et al., 2013).

\subsection{Efetividade clínica, indicações e limitações do uso do Diamino Fluoreto de Prata}

Em crianças com cárie na primeira infância, o DFP tem efetividade comprovada na paralização do processo de desenvolvimento de cárie (Chibinski et al., 2017) ou mesmo na prevenção do desenvolvimento de novas lesões como um benefício adicional à aplicação (Oliveira et al., 2019). Esses resultados são demonstrados por ensaios clínicos randomizados realizados em pré-escolares com cárie na primeira infância, como demonstrado no Quadro 1. Nessas circunstâncias, o DFP emerge como uma opção terapêutica adicional em Odontopediatria por suprir a necessidade de um grupo particular de pacientes que experimentam atividade da doença cárie, sendo, portanto, indicado globalmente como estratégia de prevenção terciária da CPI (OMS, 2017; Bavel et al., 2020).

Quadro 1: Ensaios clínicos randomizados sobre a efetividade clínica do diamino fluoreto de prata em pré-escolares com cárie na primeira infância.

\begin{tabular}{|c|c|c|}
\hline Autor, ano & Protocolo & Resultados principais \\
\hline Chu et al., 2002 & $\begin{array}{l}\text { Diamino fluoreto de prata a } 38 \% \text { aplicado uma } \\
\text { vez ao ano }\end{array}$ & $\begin{array}{l}\text { O diamino fluoreto de prata foi } 1,84 \\
\text { vezes mais eficaz na paralização da lesão } \\
\text { de cárie em dentina de dentes decíduos } \\
\text { que o verniz de fluoreto de sódio. }\end{array}$ \\
\hline Llodra et al., 2005 & $\begin{array}{l}\text { Diamino fluoreto de prata a } 38 \% \text { aplicado duas } \\
\text { vezes ao ano }\end{array}$ & $\begin{array}{l}\text { O incremento de cárie no grupo tratado } \\
\text { com diamino fluoreto de prata foi } 4,93 \\
\text { vezes menor em comparação ao grupo } \\
\text { controle (sem tratamento). }\end{array}$ \\
\hline Yee et al., 2009 & $\begin{array}{l}\text { 1. Diamino fluoreto de prata a } 38 \% \text { associado } \\
\text { ao ácido tânico aplicado uma vez ao ano, } \\
\text { 2. Diamino fluoreto de prata a } 38 \% \text { aplicado } \\
\text { uma vez ao ano, } \\
\text { 3. Diamino fluoreto de prata a } 12 \% \text { aplicado } \\
\text { uma vez ao ano. }\end{array}$ & $\begin{array}{l}\text { O diamino fluoreto de prata a } 38 \% \text {, } \\
\text { associado ou não ao ácido tânico, foi mais } \\
\text { eficaz ( } \pm 1,46 \text { vezes) que o diamino } \\
\text { fluoreto de prata a } 12 \% \text { na paralização do } \\
\text { processo de cárie em dentes decíduos. }\end{array}$ \\
\hline Braga et al., 2009 & $\begin{array}{l}\text { 1. Diamino fluoreto de prata a } 10 \% \text { aplicado } \\
\text { duas vezes no ano, em um intervalo de } 1 \\
\text { semana. } \\
\text { 2. Selante ionomérico para fóssulas e fissuras. } \\
\text { 3. Escovação dental }\end{array}$ & $\begin{array}{l}\text { O efeito do diamino fluoreto de prata é } \\
\text { significativamente maior que a escovação } \\
\text { e que o selante Ionomérico. Entretanto, } \\
\text { essa diferença é clinicamente } \\
\text { insignificante após } 18 \text { meses. }\end{array}$ \\
\hline Milgrom et al., 2017 & $\begin{array}{l}\text { 1. Diamino fluoreto de prata a } 38 \% \\
\text { 2. Placebo }\end{array}$ & $\begin{array}{l}\text { A inativação das lesões de cárie após duas } \\
\text { semanas foi significativamente maior } \\
\text { (Risco relativo: } 17,3 \text { ) no grupo tratado } \\
\text { com diamino fluoreto de prata do que no } \\
\text { grupo placebo. }\end{array}$ \\
\hline
\end{tabular}

Fonte: Autores.

As indicações do material envolvem casos específicos de alta atividade de cárie, casos em que o tratamento é desafiador do ponto de vista médico ou comportamental, e/ou casos em que as lesões cariosas não podem ser tratadas em uma 
visita, e em casos em que a criança não tem acesso à serviços odontológicos (Horst, et al., 2016). As condições clínicas de indicações e contraindicações a respeito do DFP encontram-se detalhadas no quadro 2.

Quadro 2: Indicações e contraindicações do diamino fluoreto de prata na clínica odontopediátrica.

\begin{tabular}{|l|}
\hline Indicações \\
\hline 1. \\
2. Lesões de cárie ativas (esmalte, dentina, cavitadas ou não) \\
3. Lesões não restauráveis \\
4. Lesões cariosas em qualquer superfície (radicular, oclusal, proximal, lisa) (dentes decíduos e permanentes) \\
5. Crianças com múltiplas lesões de cárie, na impossibilidade de tratamento em uma sessão \\
6. Crianças não cooperativas, ou que o comportamento / condições médicas limitem o tratamento restaurador invasivo \\
7. $\quad$ Crianças com alto risco de cárie com condições médicas ou psicológicas que limitem outras abordagens de tratamento (por \\
Cxemplo, pacientes fóbicos, ou com deficiências) \\
\hline Contraindicações \\
\hline 1. Lesões de cárie com sinais ou sintomas clínicos de pulpite irreversível ou abscesso / fístula dentária, e/ou que tenham sinais \\
2. Padiográficos de comprometimento pulpar ou patologia perirradicular \\
\hline
\end{tabular}

Fonte: Horst et al. (2016).

No cenário pandêmico, o DFP oferece uma alternativa indolor, rápida (1-4 minutos clínicos) e menos estressante para a criança e seus pais (Corrêa-Faria et al., 2020) numa época em que se evidencia o impacto emocional negativo da COVID-19 em suas vidas (Bavel et al., 2020; Fegert et al., 2020) e se faz necessária a redução do estresse e ansiedade no ambiente do consultório odontológico. É importante considerar que dentro da complexidade do atendimento odontopediátrico na primeira infância, na qual a criança pode não ter a habilidade de cooperar e a estabilização protetora é requerida, o maior contato entre os sujeitos envolvidos (criança, pais e profissional) pode elevar o risco de contaminação. Portanto, como medida compreensiva de redução da contaminação pela COVID-19 e tratamento da doença cárie, pode-se supor que o uso do DFP reduz o contato físico durante o atendimento.

Considerando o uso de instrumentos rotatórios no aumento da disseminação do vírus via aerossol e gotas de saliva e sangue (Meng et al., 2020; Luzzi et al., 2020), a ausência de necessidade de remoção de tecido cariado para atividade (e efetividade) do produto (Chu et al., 2002) assim como a possibilidade de tratar a doença cárie fora do ambiente do consultório ou com mínima contaminação são argumentos plausíveis para seu uso durante a pandemia. Ainda, baseado no seu efeito preventivo e terapêutico, o DFP pode evitar a necessidade de consultas futuras (Johhnson et al., 2019) em um cenário em que é conveniente limitar o contato com o dentista.

No contexto da saúde pública e também da iniciativa privada, o DFP é uma alternativa interessante pelo potencial para reduzir agravos em saúde bucal a um custo muito baixo (Johhnson et al., 2019) e por ser uma modalidade de tratamento simples (sem grandes necessidades de treinamento), acessível (centavos por aplicação) e não invasiva. A eficácia clínica do diamino fluoreto de prata, aliada à sua praticidade e baixo custo, torna viável sua utilização nas mais variadas situações clínicas e até mesmo em realidades com limitação de recursos e alta demanda de tratamento. As indicações e contraindicações específicas do tratamento com DFP estão sumarizadas na Tabela 1.

Uma problemática relevante ao uso clínico do diamino fluoreto de prata é o comprometimento estético causado pelo escurecimento da superfície dental afetada. Tal fato, pode diminuir a aceitação dos pais ou responsáveis na escolha da técnica, principalmente quando o tratamento envolver os dentes anteriores. Entretanto, é importante ponderar a influência do cirurgiãodentista ou odontopediatra nesse processo. No estudo de Clemens et al. (2018) foi constatado que a aceitação dos pais foi positiva desde que houvesse a explicação do porquê da escolha do material. Portanto, os pais normalmente concordam com a 
terapia ao entenderem que o tratamento é seguro, tem eficácia comprovada, é indolor e tem o potencial para evitar procedimentos mais invasivos e novas lesões (Kumar et al., 2016). Nessas circunstâncias, é importante que o cirurgião-dentista explique detalhadamente o protocolo de tratamento, elencando todos os aspectos positivos e negativos do uso do DFP. Além disso, a empatia no processo de tomada de decisão direciona a avaliação individualizada da auto percepção estética e de saúde da criança e dos pais.

\subsection{Uso clínico do Diamino Fluoreto de Prata no contexto da COVID-19}

No mercado brasileiro as formulações de diamino fluoreto de prata disponíveis para compra são o Ancárie® (Maquira, Brasil) e Cariestop ${ }^{\circledR}$ (Biodinâmica, Brasil) nas concentrações de 12 e $30 \%$ e o Riva Star® (SDI, Austrália) na concentração de $38 \%$. Tais concentraçães são em relação a quantidade de diamino fluoreto de prata a cada $100 \mathrm{~mL}$ de produto. Estudos laboratoriais e clínicos têm demonstrado que o efeito do DFP é concentração-dependente (Yee et al., 2009; Mei et al., 2012; Fung et al., 2016; Fung et al., 2018) e a melhor concentração para ser usada é a de 38\%, sendo a de $12 \%$ não efetiva no controle da cárie dentária (Chibinski et al., 2017; Mei et al., 2016; Contreras et al., 2017; Soares-Yoshikawa et al., 2020). Dessa forma, consideramos prudente para realidade brasileira o uso do DFP em concentrações iguais a 30\% ou a 38\%.

A aplicação sobre a lesão de cárie (Gao et al., 2016) seguida de uma reaplicação semestral é o protocolo que resulta em maior sucesso na paralização de lesões de cárie quando comparada a aplicação única (Zhi et al., 2012). O passo-a-passo de aplicação do DFP é descrito a seguir (Horst et al., 2016):

1. Limpar ou realizar profilaxia dentária para remoção do biofilme dental da superfície que receberá o DFP (com escova de dente e pasta profilática ou água e pedra pomes);

2. Proteção de tecidos moles (face, lábios, mucosas) com vaselina, para evitar a pigmentação das mucosas ou lesões em tecidos moles;

3. Isolamento relativo do campo operatório com roletes de algodão;

4. Agitação do frasco para homogeneização da solução;

5. Colocação de uma gota da solução em um pote Dappen de vidro (1 gota é o suficiente para aplicação em 5-6 cavidades);

6. Secagem do dente com bolinhas de algodão secas;

7. Aplicação do DFP com aplicador descartável na cavidade, e deixar agir por 1 minuto;

8. Remover o excesso do produto com algodão, gaze ou lavar a cavidade;

9. Remoção do isolamento relativo

O isolamento da gengiva e mucosa é imprescindível durante o tratamento pois o produto causa reações irritantes ao tecido mole e pode causar manchamento. No entanto, se ocorrer contato do produto com a mucosa deve-se neutralizar o efeito do DFP com solução salina a 3\%. A utilização de iodo (polvidine tópico) tem sido usada de forma empírica pelos autores e tem apresentado bom efeito na neutralização de pigmentação em tecidos moles. Além disso, quando em contato com a pele e roupas, é recomendado lavar a superfície com água, amônia, ou água oxigenada. Para evitar toxicidade sistêmica, a dose máxima recomendada por visita é de 1 gota $(25 \mu \mathrm{L}) / 10 \mathrm{~kg}$ de peso do paciente (Horst et al., 2016).

Antes da consulta, o cirurgião-dentista deve fazer uma teletriagem do paciente para verificar se há algum indício de sintoma da COVID-19 (caso não confirmado, porém suspeito) ou se há confirmação de contaminação. Pacientes confirmados com COVID-19 que estejam com a doença de forma ativa ou que estejam em recuperação com menos de 30 dias desde a recuperação total da doença são incluídos no grupo de pessoas em quarentena que devem ser atendidos somente em caso de urgência (Alharbi et al.,2020). Em respeito as normas de isolamento social, a mesma recomendação em relação a atendimentos emergenciais é direcionada para as crianças em que não apresentem sintomas da doença nem suspeita de contaminação. 
Portanto, o tratamento com DFP deverá ser aplicado durante consultas de urgência dependendo da necessidade em relação ao controle das lesões de cárie (Alharbi et al., 2020). É importante ressaltar que o cirurgião-dentista não deve negligenciar (sob qualquer argumento) a necessidade de paramentação adequada e controle de infecção no ambiente do consultório odontológico. Aconselhamos a leitura das orientações técnicas da agência nacional de vigilância sanitária para funcionamento dos serviços de saúde (ANVISA, 2020).

\section{Conclusões}

O controle de lesões de cárie em crianças com CPI se torna mais desafiador no cenário da pandemia da COVID-19, e a utilização do DFP é considerada uma opção eficaz, barata, fácil e com riscos reduzidos de infecção pela COVID-19. Entretanto, o Odontopediatra deve ponderar as condições de cada paciente e atendimento respeitando as indicações e contraindicações do tratamento, seguindo os passos clínicos e as normas de biossegurança. Assim, pesquisas bem delineadas que observem o tratamento de escolha para a CPI bem como as preferências dos Odontopediatras durante a pandemia da COVID-19 devem ser incentivadas.

Nesse contexto, a análise da literatura evidencia que a maioria dos estudos clínicos realizados investigaram os efeitos do DFP em lesões de cárie, dessa forma, no futuro, a realização de pesquisas clínicas que investiguem o efeito do DFP sobre a incidência de cárie em outros dentes quando do seu uso também devem ser estimuladas, já que a efetividade que as aplicações de DFP apresentam na prevenção da cárie na primeira infância constitui um aspecto de grande relevância.

\section{Referências}

Alharbi, A., Alharbi, S., \& Alqaidi S (2020). Guidelines for dental care provision during the COVID-19 pandemic. Saudi Dent J. 32(4),181-6.

Aquino, E. M. L., Silveira, I. H., Pescarini, J. M., et al (2020). Social distancing measures to control the COVID-19 pandemic: potential impacts and challenges in Brazil. Cien Saude Colet, 25(suppl 1), 2423-46.

Bavel, J. J. V., Baicker, K., Boggio, O. S., et al (2020). Using social and behavioural science to support COVID-19 pandemic response. Nat Hum Behav, 4(5), 460-71.

Braga, M. M., Mendes, F. M., De Benedetto, M. S., \& Imparato, J. C. (2009). Effect of silver diammine fluoride on incipient caries lesions in erupting permanent first molars: a pilot study. Journal of Dentistry for Children, 76(1),28-33.

Brasil 2020. Agência Nacional de Vigilância Sanitária (ANVISA), Nota Técnica GVIMS/GGTES/ANVISA No 04/2020. Brasília.

Casamassimo, P. S., Thikkurissy, S., Edelstein, B. L., \& Maiorini E (2009). Beyond the dmft. The human and economic cost of early childhood caries. J Am Dent Assoc, 140, 650-7.

Chibinski, A. C., Wambier, L. M., Feltrin, J., Loguercio, A. D., Wambier, D. S., \& Reis (2017). A. Silver diamine fluoride has efficacy in controlling caries progression in primary teeth: a systematic review and meta-analysis. Caries Res, 51(5), 527-4.

Chu, C. H., Lo, E. C. M., \& Lin, H. C. (2002). Effectiveness of Silver Diamine Fluoride and Sodium Fluoride Varnish in Arresting Dentin Caries in Chinese Pre-school Children. J Dent Res, 81(11), 767-770.

Chu, C. H., \& Lo, E. C. M. (2008). Promoting caries arrest in children with silver diamine fluoride: a review. Oral Health Prev Dent, 6(4), 315-321.

Chu, C. H., Lo, E. C. M., \& Lin, H. C. (2002). Effectiveness of Silver Diamine Fluoride and Sodium Fluoride Varnish in Arresting Dentin Caries in Chinese Pre-school Children. J Dent Res, 81(11), 767-70.

Clemens, J., Gold, J., \& Chaffin J (2018). Effect and acceptance of silver diamine fluoride treatment on dental caries in primary teeth. J Public Health Dent, 78(1), 63-68.

Contreras, V., Toro, M. J., \& Elías-Boneta, A. R (2017). Encarnación-Burgos A. Effectiveness of silver diamine fluoride in caries prevention and arrest: a systematic literature review. Gen Dent, 65(3), 22-29.

Corrêa-Faria, P., Viana, K. A., Raggio, D. P., Hosey, M. T., \& Costa, L. R (2020). Recommended procedures for the management of early childhood caries lesions - a scoping review by the Children Experiencing Dental Anxiety: Collaboration on Research and Education (CEDACORE). BMC Oral Health, 20(1), 75 .

de Almeida, L. F., Cavalcanti, Y. W., \& Valenca, A. (2011). In vitro antibacterial activity of silver diamine fluoride in different concentrations. Acta Odontol Latinoam, 24(2), 127-131. 
Duangthip, D., Chu, C. H., \& Lo, E. C. (2016). A randomized clinical trial on arresting dentine caries in preschool children by topical fluorides-18 month results. J Dent, 44, 57-63.

Fegert, J. M., Vitiello, B., Plener, P. L., \& Clemens, V (2020). Challenges and burden of the Coronavirus 2019 (COVID-19) pandemic for child and adolescent mental health: a narrative review to highlight clinical and research needs in the acute phase and the long return to normality. Child Adolesc Psychiatry Ment Health, 14, 20.

Fung, M. H. T., Duangthip, D., Wong, M. C. M., Lo, E. C. M., \& Chu, C. H. (2016). Arresting Dentine Caries with Different Concentration and Periodicity of Silver Diamine Fluoride. JDR Clin Trans Res, 1(2), 143-52.

Fung, M. H. T., Duangthip, D., Wong, M. C. M., Lo, E. C. M., \& Chu, C. H. (2018). Randomized Clinical Trial of $12 \%$ and $38 \%$ Silver Diamine Fluoride Treatment. J Dent Res, 97(2), 171-8.

Gao, S. S., Zhao, I. S., Hiraishi, N., Duangthip, D., Mei, M. L., Lo, E. C. M., \& Chu, C. H. (2016). Clinical trials of silver diamine fluoride in arresting caries among children: a systematic review. JDR Clin Transl Res, 1(3), 201-10.

Garrett L (2020). COVID-19: the medium is the message. Lancet, 395(10228), 942-943.

Horst, J. A., Ellenikiotis, H., \& Milgrom, P. L (2016). UCSF Protocol for Caries Arrest Using Silver Diamine Fluoride: Rationale, Indications and Consent. J Calif Dent Assoc. 44(1), 16-28.

Johhnson, B., Serban, N., Griffin, P. M., \& Tomar, S L. (2019). Projecting the economic impact of silver diamine fluoride on caries treatment expenditures and outcomes in young U.S. children. J Public Health Dent. 79(3), 215-21.

Kissler, S. M., Tedijanto, C., Goldstein, E., Grad, Y. H., \& Lipsitch, M (2020). Projecting the transmission dynamics of SARS-CoV-2 through the postpandemic period. Science, 368(6493), 860-8.

Klein, U., Kanellis, M \& Drake, D (1999). Effects of four anticaries agents on lesion depth progression in an in vitro caries model. Pediatr Dent, 21(3), 176180 .

Knight, G. M., McIntyre, J. M., Craig, G. G., Mulyani Zilm, O. S., \& Gully, N. J. (2005). An in vitro model to measure the effect of a silver fluoride and potassium iodide treatment on the permeability of demineralized dentine to Streptococcus mutans. Aust Dent J, 50(4), 242-245.

Kumar, S., Tadakamadla, J., Duraiswamy, P., \& Kulkarni, S (2016). Dental Caries and its Socio-Behavioral Predictors- An Exploratory Cross-Sectional Study. J Clin Pediatr Dent, 40(3), 186-192.

Llodra, J. C., Rodriguez, A., Ferrer, B., Menardia, V., Ramos, T., \& Morato, M (2005). Efficacy of silver diamine fluoride for caries reduction in primary teeth and first permanente molars of schoolchildren: 36-month clinical trial. J Dent Res. 84(8), 721-724.

Luzzi, V., Ierardo, G., Bossù, M., \& Polimeni, A (2020). COVID-19: Pediatric Oral Health during and after the Pandemics. Appl. Sci., 10, x.

Matrajt, L \& Leung, T (2020). Evaluating the Effectiveness of Social Distancing Interventions to Delay or Flatten the Epidemic Curve of Coronavirus Disease. Emerg Infect Dis, 26(8), 10.

Mazzoni, A., Tjäderhane, L., Checchi, V., Di Lenarda, R., Salo, T., Tay, F. R., Pashley, D. H., et al (2015). Role of dentin MMPs in caries progression and bond stability. J Dent Res, 94(2), 241-251.

Mei, M. L., Li, Q. L., Chu, C H., Yiu, C K., \& Lo, E C. (2012). The inhibitory effects of silver diamine fluoride at different concentrations on matrix metalloproteinases. Dent Mater, 28(8), 903-8.

Mei, M. L., Li, Q. L., Chu, C H., Lo, E. C., \& Samaranayake, L. P (2013). Antibacterial effects of silver diamine fluoride on multi-species cariogenic biofilm on caries. Ann Clin Microbiol Antimicrob, 12,4.

Mei, M. L., Ito, L., Cao, Y., Li, QL., Chu, C. H., \& Lo, E. C (2014). The inhibitory effects of silver diamine fluorides on cysteine cathepsins. J Dent, 42(3), $329-335$.

Mei, M. L., Lo, E. C. M., \& Chu, C. H. (2016). Clinical use of silver diamine fluoride in dental treatment. Compend Contin Educ Dent, 37(2), 93-8.

Meng, L., Hua, F \& Bian, Z (2020). Coronavirus Disease 2019 (COVID-19): Emerging and future challenges for dental and oral medicine. J Dent Res, 99, 481-7.

Milgrom, P., Horst, J. A., Ludwig, S., Rothen, M., Chaffee B. W., et al (2017). Topical Silver Diamine Fluoride for Dental Caries Arrest in Preschool Children: A Randomized Controlled Trial and Microbiological Analysis of Caries Associated Microbes and Resistance Gene Expression. J Dent, S03005712(17), 30212-30219.

Obermajer, N., Jevnikar, Z., Doljak, B., \& Kos, J (2008). Role of cysteine cathepsins in matrix degradation and cell signalling. Connect Tissue Res, 49(3), 193196.

Oliveira, B. H., Rajendra, A., Veitz-Keenan, A., \& Niederman, R (2019). The effect of silver diamine fluoride in preventing caries in the primary dentition: a systematic review and meta-analysis. Caries Res, 53(1), 24-32.

Organização Mundial da Saúde (OMS) (2020). Overview of public health and social measures in the context of COVID-19. Interim guidance. https://www.who.int/publications/i/item/overview-of-public-health-and-social-measures-in-the-context-of-covid-19.

Organização Mundial da Saúde (OMS) (2017). WHO Expert Consultation on Public Health Intervention against Early Childhood Caries: report of a meeting, Bangkok, Thailand. World Health Organization. 
Research, Society and Development, v. 10, n. 6, e7710615380, 2021

(CC BY 4.0) | ISSN 2525-3409 | DOI: http://dx.doi.org/10.33448/rsd-v10i6.15380

Pitts, N., Baez, R., Diaz-Guallory, C., et al. (2019). Early Childhood Caries: IAPD Bangkok Declaration. Int J Paediatr Dent, 29:384-386

Rosas, S. G. P., Tellez, M. A. A., \& EspinozaII, E. V. (2014). In vitro efficiency of fluoride-containing compounds on remineralization of carious enamel lesions under cyclic pH conditions. Revista Odontológica Mexicana,18(2), 96-104.

Rosenblatt, A., Stamford, T. C., \& Niederman R (2009). Silver diamine fluoride: a caries "silver-fluoride bullet". J Dent Res, 88(2),116-25.

Schwendicke, F., Splieth, C., Breschi, L., et al (2019). When to intervene in the caries process? An expert Delphi consensus statement. Clin Oral Investig, 23(10), 3691-703.

Soares-Yoshikawa, A. L., Cury, J. A., \& Tabchoury, C. O. M. (2020). Fluoride Concentration in SDF Commercial Products and Their Bioavailability with Demineralized Dentine. Braz Dent J, 31(3), 257-263.

Suzuk,i T., Tsutsum, N., Sobue, S et al (1976). Effect of diammine silver fluoride on plaque formation by Streptococcus mutans 2. Mode of action of plaque inhibition. Jpn J Oral Biol, 18, 268-278.

Suzuki, T., Nishida, M., Sobue, S., \& Moriwaki, Y (1974). Effects of diammine silver fluoride on tooth enamel. J Osaka Univ Dent Sch, 14, 61-72.

Tinanoff, N., Baez, R. J., Diaz Guillory, C., Donly, K. J., Feldens, C. A., McGrath, C., Phantumvanit, P., Pitts, N. B., Seow, W. K., Sharkov, N., Songpaisan, Y., \& Twetman, S (2019). Early childhood caries epidemiology, aetiology, risk assessment, societal burden, management, education, and policy: Global perspective. Int J Paediatr Dent, 29(3), 238-48.

Yee, R., Holmgren, C., Mulder, J., Lama, D., Walker, D., \& van Palenstein Helderman, W (2009). Efficacy of silver diamine fluoride for Arresting Caries Treatment. J Dent Res, 88(7), 644-7.

Zhao, I. S., Gao, S. S., Hiraishi, N., Burrow, M. F., Duangthip, D., Mei, M. L., et al (2018). Mechanisms of silver diamine fluoride on arresting caries: a literature review. Int Dent J, 68(2), 67-76.

Zhi, Q. H., Lo, E. C., \& Lin, H. C (2012). Randomized clinical trial on effectiveness of silver diamine fluoride and glass ionomer in arresting dentine caries in preschool children. $J$ Dent, 40(11), 962-7. 